\title{
Correspondence
}

Br Heart $\mathcal{F} 1980$; 44: 724

\section{Massive dilatation of the left atrium}

Sir,

It is accepted that left recurrent laryngeal nerve paralysis in association with massive dilatation of the left atrium is caused by compression between the aortic arch and pulmonary artery by upward displacement of the latter.

The cause of dysphagia in these circumstances is less clear. Morgan and Mourant ${ }^{1}$ suggest that it is the result of disordered peristalsis consequent upon stretching of autonomic nerve fibres between the posterior pulmonary plexuses and the oesophagus with possible damage to nerves supplying the oesophageal plexus. This is an ingenious explanation. Five of our 15 patients with massive atrial dilatation complained of difficulty in swallowing solid food and two of these also complained of difficulty in swallowing liquids. ${ }^{2}$ One had painful dysphagia after swallowing a large bolus. Two patients pointed to the site of the obstruction at the suprasternal notch and the xiphisternum, respectively, whereas in all, fluoroscopy after a barium swallow showed the obstruction to be a few centimetres below the tracheal bifurcation.
While increased left atrial pressure in mitral valve disease is not likely to exceed peak intraoesophageal pressure during active peristalsis, the displacement and narrowing of the oesophagus is considered sufficient to lead to dysphagia. In addition, widening of the carinal angle has led to oesphageal indentation by the left main bronchus.

Most patients with massive atrial dilatation are in atrial fibrillation, but even in the absence of a normally pulsating atrium the pressure exerted is sufficient to erode vertebrae.

Raymond Daley,
The White House,
The Green,
Woodchurch,
Ashford, Kent.

\section{References}

1 Morgan AA, Mourant AJ. Left vocal cord paralysis and dysphagia in mitral valve disease. $B r$ Heart $\mathcal{f}$ 1980 ; 43: 470-3.

2 Daley R, Franks R. Massive dilatation of the left auricle. $Q f$ Med 1949; NS 18: 81-91. 